Chirurg 2013 $\cdot 84: 700$

DOI 10.1007/s00104-013-2567-z

Online publiziert: 22. Juni 2013

(c) Springer-Verlag Berlin Heidelberg 2013

O. Strobel · M.W. Büchler

Klinik für Allgemein-, Viszeral- und Transplantationschirurgie, Universität Heidelberg

\title{
Ernährungszustand und Lebensqualität nach partieller Pankreatektomie
}

rin, Parameter der endokrinen Funktion, die Stuhlelastase sowie die Lebensqualität (Erfassungsbogen EORTC QLQ-C30) wurden prospektiv direkt präoperativ, bei Entlassung sowie 3, 6 und 12 Monate postoperativ erfasst.

\section{Ergebnisse}

Trotz unzureichender Datenlage wird allgemein angenommen, dass es nach partieller Pankreasresektion (Pankreatoduodenektomie, Pankreaslinksresektion) durch den Parenchymverlust zu einer bleibenden Einschränkung der endokrinen und exokrinen Funktion mit Reduktion des Ernährungszustands und der Lebensqualität kommt. Die Literatur beschränkt sich auf wenige Studien zum Vergleich unterschiedlicher Operationstechniken, in denen Funktion und Lebensqualität meist als sekundäre Endpunkte untersucht wurden. Prospektive, longitudinale Studien zur Entwicklung der Organfunktion, des Ernährungsstatus und der Lebensqualität nach partieller Pankreasresektion fehlten bisher, sind aber insbesondere angesichts der Ausweitung der Operationsindikationen auf benigne und prämaligne Läsionen wichtig. Park et al. führten nun eine entsprechende Studie durch.

\section{Methoden}

Von 212 Patienten mit geplanter Pankreatektomie wurden 136 Patienten in die Analyse eingeschlossen. Ausgeschlossen wurden u. a. Patienten mit Malignom und Rezidiv während des Follow-up. Das relative Körpergewicht, die Trizepshautfaltendicke, Serumprotein, Albumin, Transfer-
Insgesamt 107 Patienten erhielten eine Pankreatoduodenektomie, 29 eine Pankreaslinksresektion. Das mittlere Alter betrug 59 Jahre, das mittlere Körpergewicht direkt präoperativ $60,4 \mathrm{~kg}$ (BMI: $\left.23,1 \mathrm{~kg} / \mathrm{m}^{2}\right) .83$ Patienten (61\%) hatten ein Malignom. Die Morbidität betrug 50,7\%. Die meisten Parameter waren bei Entlassung im Vergleich zum präoperativen Wert signifikant schlechter, erholten sich aber im weiteren postoperativen Verlauf wieder. Das relative Körpergewicht und die Trizepshautfaltendicke erholten sich bis 12 Monate postoperativ auf etwa $90 \%$ des präoperativen Wertes, während das Serumprotein, Albumin und Transferrin bereits nach 3 Monaten wieder auf dem präoperativen Niveau lagen. Von 76 Patienten, bei denen präoperativ kein Diabetes vorlag, hatten 22 (29\%) nach 6 Monaten und nur noch 13 (17\%) nach 12 Monaten einen Diabetes mellitus. Nur 4 (5\%) Patienten entwickelten einen insulinpflichtigen Diabetes. Die Häufigkeit von Diarrhö und Steatorrhö näherte sich ab 3 Monaten den präoperativen Werten an, während die Stuhlelastase innerhalb des Untersuchungszeitraums signifikant erniedrigt blieb. Die Lebensqualität erreichte bereits 3 Monate postoperativ annähernd die präoperativen Werte. Insgesamt erholten sich Ernährungszustand, endokrine Funktion und Lebensqualität bei $46 \%, 53 \%$ und $57 \%$ der Patienten innerhalb der ersten 6 Monate auf präoperative Werte. In einer multivariaten Analyse wurden ein Alter ab 60 Jahre, eine Pankreatoduodenektomie, eine chronische Pankreatitis und ein Malignom als Risikofaktoren für ein schlechteres funktionelles Outcome identifiziert.

\section{Diskussion}

Die Autoren folgern, dass etwa 50\% der Patienten nach partieller Pankreatektomie innerhalb von 6 Monaten mit einer Erholung der Organfunktion, des Ernährungszustands und der Lebensqualität rechnen können. Patienten mit den identifizierten Risikofaktoren sollten einem intensivierten Follow-up und einer supportiven Therapie zugeführt werden. Wichtig ist, dass die Studie insbesondere für Patienten mit benignen und prämalignen Läsionen ein sehr gutes funktionelles Outcome mit normaler Lebensqualität zeigt. Ungeklärt bleibt, ob die an einem asiatischen Kollektiv erhobenen Ergebnisse auf westliche Patienten übertragbar sind. Hier sind weitere Studien notwendig.

\section{Korrespondenzadresse}

PD Dr. O. Strobel

Klinik für Allgemein-, Viszeral- und Transplantationschirurgie,

Universität Heidelberg,

Im Neuenheimer Feld 110, 69120 Heidelberg

Oliver.Strobel@med.uni-heidelberg.de

Interessenkonflikt. Der korrespondierende Autor gibt für sich und seinen Koautor an, dass kein Interessenkonflikt besteht. 